\title{
An Improved Collocation Approach of Euler Polynomials Connected with Bernoulli Ones for Solving Predator-Prey Models with Time Lag
}

\author{
Behrooz Basirat $(\mathbb{D}$ ) and Hamid Reza Elahi $i$ \\ Department of Mathematics, Birjand Branch, Islamic Azad University, Birjand, Iran \\ Correspondence should be addressed to Behrooz Basirat; behrooz.basirat@iaubir.ac.ir
}

Received 1 September 2019; Revised 6 November 2019; Accepted 12 December 2019; Published 1 April 2020

Academic Editor: Patricia J. Y. Wong

Copyright (C) 2020 Behrooz Basirat and Hamid Reza Elahi. This is an open access article distributed under the Creative Commons Attribution License, which permits unrestricted use, distribution, and reproduction in any medium, provided the original work is properly cited.

\begin{abstract}
This paper deals with an approach to obtaining the numerical solution of the Lotka-Volterra predator-prey models with discrete delay using Euler polynomials connected with Bernoulli ones. By using the Euler polynomials connected with Bernoulli ones and collocation points, this method transforms the predator-prey model into a matrix equation. The main characteristic of this approach is that it reduces the predator-prey model to a system of algebraic equations, which greatly simplifies the problem. For these models, the explicit formula determining the stability and the direction is given. Numerical examples illustrate the reliability and efficiency of the proposed scheme.
\end{abstract}

\section{Introduction}

In recent years, population models in various fields of mathematical biology have been proposed and studied extensively. Predator-prey interaction is the fundamental structure in population dynamics. Understanding the dynamics of predator-prey models will be very helpful for investigating multiple species interactions [1-6]. The first predator-prey model with delay was proposed by Volterra and Brelot [7]. Let $p_{1}(t)$ and $p_{2}(t)$ denote the population density of prey and predator at time $t$. In this work, we first, consider the modified version of the predator-prey model with delay [8]:

$$
\left\{\begin{array}{l}
p_{1}^{\prime}(t)=p_{1}(t)\left[r_{1}-a_{11} p_{1}(t)-a_{12} \int_{-\infty}^{t} F(t-s) p_{2}(s) \mathrm{d} s\right], \\
p_{2}^{\prime}(t)=p_{2}(t)\left[-r_{2}+a_{21} \int_{\infty}^{t} G(t-s) p_{1}(s) \mathrm{d} s-a_{22} p_{2}(t)\right],
\end{array}\right.
$$

where $r_{1}>0$ is the growth rate of the prey in the absence of predators, $a_{11}>0$ denotes the self-regulation constant of the prey, $a_{12}>0$ describes the predation of the prey by predators, $r_{2}>0$ is the death rate of predators in the absence of the prey, $a_{21}>0$ is the conversion rate for the predators, and $a_{22} \geq 0$ describes the transpacific competition among predators. $F(s)$ and $G(s)$ are nonnegative continuous delay kernels defined and integrable on $[0, \infty)$, which weigh the contribution of the predation occurred in the past to the change rate of the prey and predators, respectively. Detailed study on stability and bifurcation of system (1) can be found in Cushing [9]. When $F(t-s)=\delta\left(t-s-\tau_{1}\right)$ and $G(t-s)$ $=\delta\left(t-s-\tau_{2}\right)$-where $\delta$ is the delta function and $\tau_{1} \geq 0$ and $\tau_{2} \geq 0$ are the hunting delay and the maturation time of the 
prey species, respectively-system (1) reduces to the following Lotka-Volterra predator-prey model with two discrete delays:

$$
\left\{\begin{array}{l}
p_{1}^{\prime}(t)=p_{1}(t)\left[r_{1}-a_{11} p_{1}(t)-a_{12} p_{2}\left(t-\tau_{1}\right)\right] \\
p_{2}^{\prime}(t)=p_{2}(t)\left[-r_{2}+a_{21} p_{1}\left(t-\tau_{2}\right)-a_{22} p_{2}(t)\right]
\end{array}\right.
$$

We will find the approximate solutions of the system (2) with initial conditions:

$$
\begin{aligned}
& p_{1}(0)=\alpha_{1}, \\
& p_{2}(0)=\alpha_{2},
\end{aligned}
$$

where $\alpha_{1}$ and $\alpha_{2}$ are positive numbers.

The exact solution for the predator-prey models in the general form does not exist; therefore, numerical methods are needed to computing the population densities of the prey and predator. Some methods [10-12] are proposed to handle an approximate solution of the predator-prey system, such as finite-difference, finite element, and spectral methods.

The organization of this paper is as follows. In Section 2, we study the stability of the predator-prey model. In Section 3, matrix relations for Euler polynomials connected with Bernoulli ones will be discussed. In Section 4, we shall present our method in detail. Finally, we give a few numerical examples in Section 5.

\section{Stability}

In this section, we review some results on the stability of the predator-prey model. To begin with, we first consider system (2) appearing in the interspecific interaction terms of both equations:

$$
\left\{\begin{array}{l}
p_{1}^{\prime}(t)=p_{1}(t) f\left(p_{1}(t), p_{2}\left(t-\tau_{1}\right)\right) \\
p_{2}^{\prime}(t)=p_{2}(t) g\left(p_{1}\left(t-\tau_{2}\right), p_{2}(t)\right)
\end{array}\right.
$$

where $\tau_{i} \geq 0, i=1,2$, is a constant. Denote $C=C([-\tau$, $0] \times R)$, where $\tau=\max \left\{\tau_{1}, \tau_{2}\right\}$. Assume that $f: R \times C \longrightarrow R$ and $g: R \times C \longrightarrow R$ satisfy the following assumptions:

$\left(S_{1}\right)$ : there exists a point $\left(p_{1}^{*}, p_{2}^{*}\right)$ with $p_{1}^{*}, p_{2}^{*}>0$ for which $f\left(p_{1}^{*}, p_{2}^{*}\right)=g\left(p_{1}^{*}, p_{2}^{*}\right)=0$,

$\left(S_{2}\right): f$ and $g$ are continuously differentiable such that $\partial f / \partial p_{1}<0, \partial f / \partial p_{2}<0, \partial g / \partial p_{1}>0$, and $\partial g / \partial p_{2}>0$.

Assumption $\left(S_{1}\right)$ ensures that $\left(p_{1}^{*}, p_{2}^{*}\right)$ is a positive equilibrium point of system (4). Let $\phi(t)=p_{1}(t)-p_{1}^{*}$ and $\psi(t)=p_{2}(t)-p_{2}^{*}$. Then, the linearized system at $\left(p_{1}^{*}, p_{2}^{*}\right)$ is

$$
\left\{\begin{array}{l}
\phi^{\prime}(t)=\left(\frac{\partial f}{\partial p_{1}}\left(p_{1}^{*}, p_{2}^{*}\right)\right)\left(p_{1}^{*}\right) \phi(t)+\left(\frac{\partial f}{\partial p_{2}}\left(p_{1}^{*}, p_{2}^{*}\right)\right)\left(p_{1}^{*}\right) \psi\left(t-\tau_{1}\right) \\
\psi^{\prime}(t)=\left(\frac{\partial g}{\partial p_{1}}\left(p_{1}^{*}, p_{2}^{*}\right)\right)\left(p_{2}^{*}\right) \phi\left(t-\tau_{2}\right)+\left(\frac{\partial g}{\partial p_{2}}\left(p_{1}^{*}, p_{2}^{*}\right)\right)\left(p_{2}^{*}\right) \psi(t) .
\end{array}\right.
$$

Therefore, we have the following theorem.

Theorem 1. Suppose that $f$ and $g$ satisfy the assumptions $\left(S_{1}\right)$ and $\left(S_{2}\right)$. Define

$$
\begin{aligned}
a & =\frac{\partial f}{\partial p_{1}}\left(p_{1}^{*}, p_{2}^{*}\right), \\
b & =\frac{\partial f}{\partial p_{2}}\left(p_{1}^{*}, p_{2}^{*}\right), \\
c & =\frac{\partial g}{\partial p_{1}}\left(p_{1}^{*}, p_{2}^{*}\right), \\
d & =\frac{\partial g}{\partial p_{2}}\left(p_{1}^{*}, p_{2}^{*}\right) .
\end{aligned}
$$

Then, the positive equilibrium $\left(p_{1}^{*}, p_{2}^{*}\right)$ of the delayed predator-prey system (4) is absolutely stable if and only if $a \quad d+b c>0$.
For proof, see [13].

By Theorem 1, we have the following condition. If

$$
r_{1} a_{21}-r_{2} a_{11}>0
$$

then system (2) has a positive equilibrium $E^{*}=\left(p_{1}^{*}, p_{2}^{*}\right)$, where

$$
\begin{aligned}
& p_{1}^{*}=\frac{r_{1} a_{22}+r_{2} a_{12}}{a_{11} a_{22}+a_{12} a_{21}}, \\
& p_{2}^{*}=\frac{r_{1} a_{21}-r_{2} a_{11}}{a_{11} a_{22}+a_{12} a_{21}} .
\end{aligned}
$$

The condition $a \quad d+b c>0$ becomes $a_{11} a_{22}-a_{12} a_{21}>0$. Thus, by Theorem 1, we have the following result on the stability of $E^{*}=\left(p_{1}^{*}, p_{2}^{*}\right)$.

Corollary 1. If condition (7) is satisfied, i.e., if the positive equilibrium $E^{*}=\left(p_{1}^{*}, p_{2}^{*}\right)$ of system (2) exists, then it is absolutely stable if and only if $a_{11} a_{22}-a_{12} a_{21}>0$. 
He [14] and Lu and Wang [15] showed that the positive equilibrium $E^{*}=\left(p_{1}^{*}, p_{2}^{*}\right)$ of system (2) is globally stable. The above stability result depends on the assumption that $a_{11} a_{22}-a_{12} a_{21}>0$. If $a_{11} a_{22}-a_{12} a_{21}<0$, then Faria [16] proved the following result.

Corollary 2. If $a_{11} a_{22}-a_{12} a_{21}<0$ and $a_{11} a_{22} \neq 0$, then there is a critical value $\tau_{2,0}>0$, such that $E^{*}$ of system (2) is asymptotically stable when $\tau_{2}<\tau_{2,0}$ and unstable when $\tau_{2}>\tau_{2,0}$.

\section{Matrix Relations for Euler Polynomials}

Bernoulli polynomials $B_{n}(x)$ are usually defined from the generating function for $|t|<2 \pi$ :

$$
G(x, t):=\frac{t e^{x t}}{e^{t}-1}=\sum_{n=0}^{\infty} B_{n}(x) \frac{t^{n}}{n !},
$$

and Bernoulli numbers $\beta_{n}$ : $=B_{n}(0)$ are contained in many mathematical formulas such as the Taylor expansion in a neighborhood of the origin of trigonometric and hyperbolic tangent and cotangent functions, and they can be obtained by the generating function [17]:

$$
\frac{t}{e^{t}-1}=\sum_{n=0}^{\infty} \beta_{n} \frac{t^{n}}{n !}
$$

Euler studied Bernoulli polynomials, and these polynomials are employed in the integral representation of differentiable periodic functions and play an important role in the approximation of such functions using polynomials. Many early Euler and Bernoulli polynomial implementations can be contained in [18-20]. Euler polynomials are strictly related to Bernoulli and are used in Taylor's expansion in the trigonometric and hyperbolic secant function districts. Recursive computation of Bernoulli and Euler polynomials can be obtained by using the following formulas:

$$
\begin{array}{r}
\sum_{k=0}^{n-1}\left(\begin{array}{l}
n \\
k
\end{array}\right) B_{k}(t)=n t^{n-1}, \quad n=2,3, \ldots, \\
E_{n}(t)+\sum_{k=0}^{n}\left(\begin{array}{l}
n \\
k
\end{array}\right) E_{k}(t)=2 t^{n}, \quad n=1,2, \ldots
\end{array}
$$

Euler polynomials $E_{n}(t)$ can also be defined as polynomials of degree $n \geq 0$ satisfying the conditions:

(1) $E_{m}^{\prime}(t)=m E_{m-1}(t), m \geq 1$

(2) $E_{m}(t+1)+E_{m}(t)=2 t^{m}, m \geq 1$
The above conditions express a recurrence relation to Euler polynomials. An explicit formula for $E_{n}(t)$ is represented as

$$
E_{n}(t)=\frac{1}{n+1} \sum_{k=1}^{n+1}\left(2-2^{k+1}\right)\left(\begin{array}{c}
n+1 \\
k
\end{array}\right) \beta_{k} t^{n+1-k},
$$

where $\beta_{k}=B_{k}(0)$ is the first Bernoulli numbers for each $k=$ $0,1, \ldots, n[21]$.

3.1. Function Approximation. Let $H=L^{2}[0,1]$, and assume that $\left\{E_{0}(t), E_{1}(t), \ldots, E_{N}(t)\right\}$ be the set of Euler polynomials, and $P_{H}=\operatorname{span}\left\{E_{0}(t), E_{1}(t), \ldots, E_{N}(t)\right\}$, and $h(t)$ be an arbitrary element in $H$. Since $P_{H}$ is a finite dimensional vector space, $h(t)$ has the unique best approximation out of $P_{H}$ such as $\widetilde{p}(t)$, that is,

$$
\|h(t)-\widetilde{p}(t)\| \leq\|h(t)-p(t)\|, \quad \forall p(t) \in P_{H} .
$$

Since $\widetilde{p}(t) \in P_{H}$, there exist unique coefficients $p_{0}, p_{1}, \ldots, p_{N}$, such that

$$
\left\{\begin{array}{l}
h(t) \simeq \widetilde{p}(t)=\sum_{n=0}^{N} p_{n} E_{n}(t)=P E(t), \\
h^{\prime}(t) \simeq \widetilde{p}^{\prime}(t)=\sum_{n=0}^{N} p_{n} E_{n}^{\prime}(t)=P E^{\prime}(t),
\end{array}\right.
$$

where

$$
\begin{aligned}
P & =\left[p_{0}, p_{1}, \ldots, p_{N}\right], \\
E(t) & =\left[E_{0}(t), E_{1}(t), \ldots, E_{N}(t)\right]^{T}, \\
E^{\prime}(t) & =D E(t),
\end{aligned}
$$

such that

$$
D=\left[\begin{array}{ccccc}
0 & 0 & \cdots & 0 & 0 \\
1 & 0 & \cdots & 0 & 0 \\
0 & 2 & \cdots & 0 & 0 \\
\vdots & \vdots & \ddots & \vdots & \vdots \\
0 & 0 & \cdots & N & 0
\end{array}\right] .
$$

Note that, from property (12) of Euler polynomials, we can represent $E(t)$ in a matrix form as follows:

$$
E(t)=M \mathscr{T}(t)
$$

where 


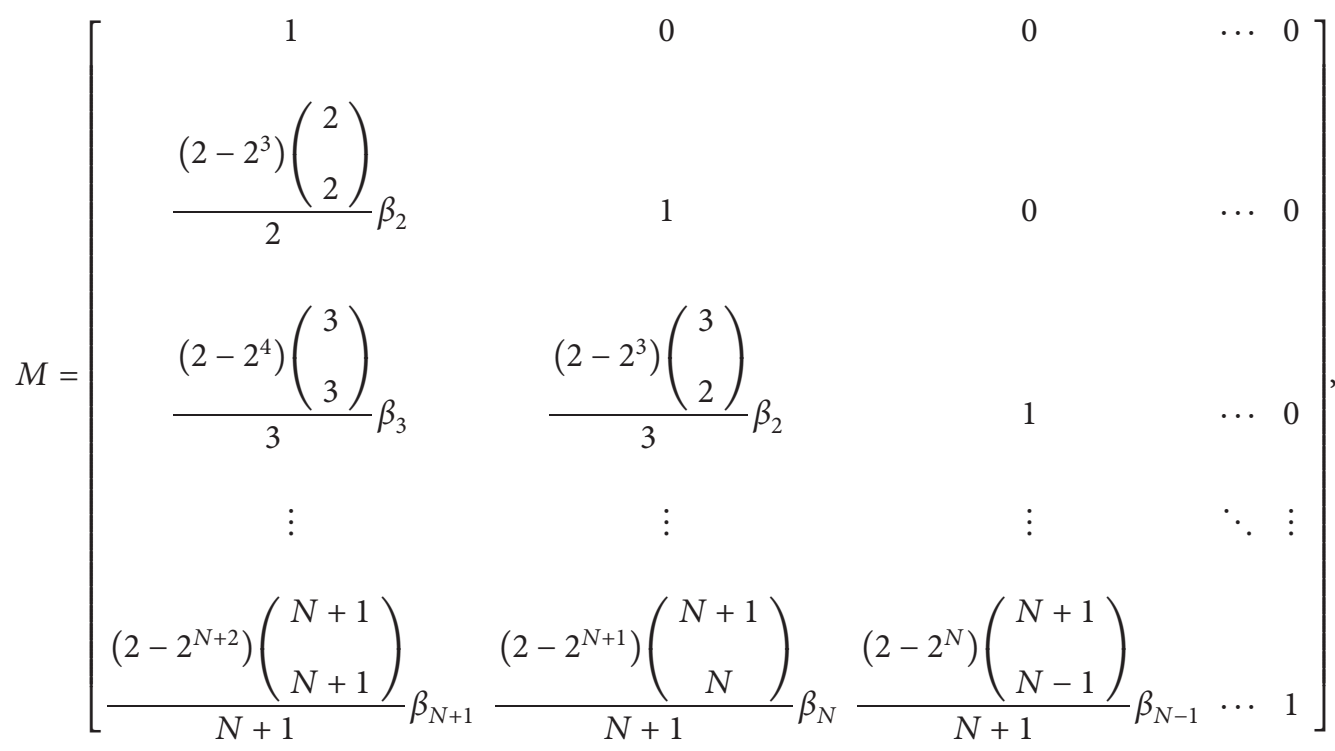

is an $(N+1) \times(N+1)$ matrix, and $\mathscr{T}(t)=$ $\left[1, t, t^{2}, \ldots, t^{N}\right]^{T}$.

\section{Numerical Solution of the Lotka-Voltra Predator-Prey Models with Discreet Delay Using Euler Operational Matrices}

Consider the Lotka-Voltra predator-prey models with discreet delay (2). For implementing the Euler operational matrix on the predator-prey model, we first approximate $p_{i}(t)$ and $p_{i}^{\prime}(t)$, for $i=1,2$, by equations (14)-(17), as follows:

$$
\left\{\begin{array}{l}
p_{i}(t) \simeq P_{i} E(t)=P_{i} M \mathscr{T}(t), \\
p_{i}^{\prime}(t) \simeq P_{i} E^{\prime}(t)=P_{i} D E(t)=P_{i} D M \mathscr{T}(t),
\end{array}\right.
$$

where $P_{i}$ is the unknown $(N+1)$ vector of coefficients.

Remark 1. Recall that the Hadamard product of two matrices, say $A$ and $B$, of the same dimension $m \times n$, is denoted by $A \circ B$, defined to be an $m \times n$-matrix, in which the $(i, j)^{\text {th }}$ entry is equal to the product of $(i, j)^{\text {th }}$ entries of $A$ and $B$, i.e., $(A \circ B)_{i, j}=A_{i, j} B_{i, j}$.

Bearing in mind the previous remark, we can simplify the delay part of system (2) to the matrix form as

$$
E(t-\tau)=M \mathscr{T}(t-\tau)=M B_{\tau} \mathscr{T}(t),
$$

where $B_{0}=I$ and for any $\tau \neq 0, B_{\tau}$ is the Hadamard product $C \circ G$ (i.e., the product of entry by entry....) such that for $1 \leq j \leq i \leq N+1, C_{i, j}=\left(\begin{array}{c}i-1 \\ j-1\end{array}\right)$ and $G_{i, j}=(-\tau)^{i-j}$, and for $i<j, C_{i, j}=G_{i, j}=0$. In other words, we may evaluate entries of $B_{\tau}$ as follows:

$$
B_{\tau}(i, j)= \begin{cases}\left(\begin{array}{l}
i-1 \\
j-1
\end{array}\right)(-\tau)^{i-j}, & i \geq j, \\
0, & i<j .\end{cases}
$$

Remark 2. Note that If $A=\left[a_{i j}\right]_{m \times n}$ and $B_{p \times q}$ are two matrices, then the Kronecker product $A \otimes B$ is defined to be the following $m p \times n q$ block matrix:

$$
A \otimes B=\left[\begin{array}{ccc}
a_{11} B & \cdots & a_{1 n} B \\
\vdots & \ddots & \vdots \\
a_{m 1} B & \cdots & a_{m n} B
\end{array}\right] .
$$

By the mixed-product property of a Kronecker product, we know that for matrices $A, B, C$, and $D$, which are of the size that matrix products $A C$ and $B \quad D$ are well-defined, the following relation holds true:

$$
(A C) \otimes(B D)=(A \otimes B)(C \otimes D) .
$$

Now, we have to estimate all expressions consisting the products of $p_{1}\left(t-\tau_{2}\right)$ and $p_{2}\left(t-\tau_{1}\right)$, existing in system (2) (note that, for $\tau_{k}=0$, the products $p_{i}\left(t-\tau_{k}\right) p_{j}(t)$, $p_{i}(t) p_{j}\left(t-\tau_{k}\right)$, and $p_{i}(t) p_{j}(t)$ can be approximated in matrix form, where $i, j \in\{1,2\})$. In order to do this, let $\{i, j\} \subseteq\{1,2\}, \quad i^{\prime}=3-i$, and $j^{\prime}=3-j$. Then, by using equations (19) and (20), we have

$$
\begin{aligned}
p_{i}( & \left.t-\tau_{i^{\prime}}\right) p_{j}\left(t-\tau_{j^{\prime}}\right) \simeq\left(P_{i} E\left(t-\tau_{i^{\prime}}\right)\right)\left(P_{j} E\left(t-\tau_{j^{\prime}}\right)\right) \\
= & \left(P_{i} M B_{\tau_{i^{\prime}}} \mathscr{T}(t)\right) \otimes\left(P_{j} M B_{\tau_{j^{\prime}}} \mathscr{T}(t)\right) \\
& =\left(P_{i} \otimes P_{j}\right)(M \otimes M)\left(B_{\tau_{i^{\prime}}} \otimes B_{\tau_{j^{\prime}}}\right)(\mathscr{T}(t) \otimes \mathscr{T}(t)) \\
& =\mathbf{P}_{i, j} \mathbf{M B}\left(\tau_{i^{\prime}}, \tau_{j^{\prime}}\right) \mathbf{T}(t),
\end{aligned}
$$

where

$$
\left\{\begin{array}{l}
\mathbf{P}_{i, j}=P_{i} \otimes P_{j}, \\
\mathbf{M}=M \otimes M, \\
\mathbf{B}\left(\tau_{i^{\prime}}, \tau_{j^{\prime}}\right)=B_{\tau_{i^{\prime}}} \otimes B_{\tau_{j^{\prime}}}, \\
\mathbf{T}(t)=\mathscr{T}(t) \otimes \mathscr{T}(t) .
\end{array}\right.
$$


With the above notations, we can rewrite system (2) as

$$
\left\{\begin{array}{l}
P_{1} D M \mathscr{T}(t)=r_{1} P_{1} M \mathscr{T}(t)-a_{11} \mathbf{P}_{1,1} \mathbf{M T}(t)-a_{12} \mathbf{P}_{1,2} \mathbf{M B}\left(0, \tau_{1}\right) \mathbf{T}(t), \\
P_{2} D M \mathscr{T}(t)=-r_{2} P_{2} M \mathscr{T}(t)+a_{21} \mathbf{P}_{2,1} \mathbf{M B}\left(0, \tau_{2}\right) \mathbf{T}(t)-a_{22} \mathbf{P}_{2,2} \mathbf{M T}(t) .
\end{array}\right.
$$

Suppose that

$$
\begin{aligned}
M_{1}(t) & =D M \mathscr{T}(t)-r_{1} M \mathscr{T}(t) \\
M_{2}(t) & =D M \mathscr{T}(t)+r_{2} M \mathscr{T}(t), \\
\mathrm{A}_{1}(t) & =a_{12} \mathbf{M B}\left(0, \tau_{1}\right) \mathbf{T}(t) \\
\mathrm{A}_{2}(t) & =-a_{21} \mathbf{M B}\left(0, \tau_{2}\right) \mathbf{T}(t), \\
\mathrm{C}_{1}(t) & =a_{11} \mathbf{M T}(t), \\
\mathrm{C}_{2}(t) & =a_{22} \operatorname{MT}(t)
\end{aligned}
$$

Then, the fundamental matrix equations of system (2) can be written in the following form:

$$
\mathbf{P} M(t)+\bar{P} A(t)+\widehat{\mathbf{P}} C(t)=\mathbf{f}(t),
$$

where

$$
\begin{aligned}
\mathbf{P} & =\left[P_{1}, P_{2}\right], \\
M(t) & =\left[\begin{array}{cc}
M_{1}(t) & 0 \\
0 & M_{2}(t)
\end{array}\right], \\
\bar{P} & =\left[\begin{array}{ll}
\mathbf{P}_{1,2}, \mathbf{P}_{2,1}
\end{array}\right], \\
A(t) & =\left[\begin{array}{cc}
A_{1}(t) & 0 \\
0 & A_{2}(t)
\end{array}\right], \\
\widehat{P} & =\left[\begin{array}{ll}
\mathbf{P}_{1,1}, \mathbf{P}_{2,2}
\end{array}\right], \\
C(t) & =\left[\begin{array}{cc}
C_{1}(t) & 0 \\
0 & C_{2}(t)
\end{array}\right], \\
\mathbf{f}(t) & =[0,0]_{1 \times 2} .
\end{aligned}
$$

4.1. Implementing the Collocation Method. Equation (28) gives us 2 nonlinear equations. Since the number of unknowns for each vector $P_{1}$ and $P_{2}$ in $(15)$ is $(N+1)$ and our system has 4 equations, the total number of unknowns is $2(N+1)$; then, we collocate equation (28) at $N$ Newton-Cotes points given as

$$
t_{s}=\frac{b}{N} s, \quad s=1,2, \ldots, N,
$$

and hence we have equation (28) and initial conditions:

$$
\left\{\begin{array}{l}
\mathbf{P} M\left(t_{s}\right)+\bar{P} A\left(t_{s}\right)+\widehat{\mathbf{P}} C\left(t_{s}\right)=\mathbf{f}\left(t_{s}\right) ; \quad s=1,2, \ldots, N \\
P_{1} M \mathscr{T}(0)=\alpha_{1}, \\
P_{2} M \mathscr{T}(0)=\alpha_{2} .
\end{array}\right.
$$

After solving linear system (31), we get $P_{1}$ and $P_{2}$. For collocating equation (31), we have used the Newton-Cotes points because of their simplicity and their good utility in our implementation regarding the speed and accuracy of answers. However, we could use other points such as the Gauss points, Clenshaw-Curtis points, and Lobatto points.

\section{Numerical Examples}

To test the method in terms of its precision and efficacy, we turn our attention to show three numerical performance results. Analytic approximate solutions of high-order accuracy are presented in most of the cases. Throughout the calculations, the absolute error is defined by

$$
\operatorname{Error}(t)=\left|p_{i}(t)-y_{1}(t)\right|,
$$

and maximum absolute error is defined by

$$
\text { Error }=\operatorname{Max}\left\{\left|p_{i}\left(t_{i}\right)-y_{1}\left(t_{i}\right)\right| ; t_{i}=\frac{i}{1000}, i=1,2, . ., 1000\right\} \text {. }
$$

The computations associated with these examples were performed using MATLAB.

Since the truncated series (17) is approximate solutions of system (31), when the function $p_{1, N}(t), p_{2, N}(t)$ and its derivatives are substituted in system (2), the resulting equation must be satisfied approximately; that is, for $t_{s}, s=1,2, \ldots, N$,

$$
\left\{\begin{array}{l}
R_{1, N}\left(t_{s}\right)=\left|p_{1, N}^{\prime}\left(t_{s}\right)-p_{1, N}\left(t_{s}\right)\left[1-p_{2, N}\left(t_{s}\right)\right]\right| \cong 0 \\
R_{2, N}\left(t_{s}\right)=\left|p_{2, N}^{\prime}\left(t_{s}\right)-p_{2, N}\left(t_{s}\right)\left[-1+p_{1, N}\left(t_{s}\right)\right]\right| \cong 0
\end{array}\right.
$$

and $\operatorname{Max}\left\{\left|R_{1, N}\left(t_{s}\right)-R_{2, N}\left(t_{s}\right)\right| \leq 10^{-r_{s}},\left(r_{s}\right.\right.$ positive integer $)$. If $\max 10^{-r_{s}}=10^{-r}$ ( $r$ positive integer) is prescribed, then the truncation limit $N$ is increased until the difference $R_{i, N}\left(t_{s}\right)$ at each of the points becomes smaller than the prescribed $10^{-r}$. All computations were carried out using MATLAB.

Example 1. We consider the following Lotka-Volterra predator-prey model with delays:

$$
\left\{\begin{array}{l}
p_{1}^{\prime}(t)=p_{1}(t)\left[1-p_{1}(t)-p_{2}(t-1.7)\right] \\
p_{2}^{\prime}(t)=p_{2}(t)\left[-1+2 p_{1}(t-1.8)-p_{2}(t)\right]
\end{array}\right.
$$

which has also a positive equilibrium $E^{*}=\left(p_{1}^{*}=\right.$ $\left.(2 / 3), p_{2}^{*}=(1 / 3)\right)[22]$. The numerical simulations for Example 1 are shown in Figures 1 and 2 . 


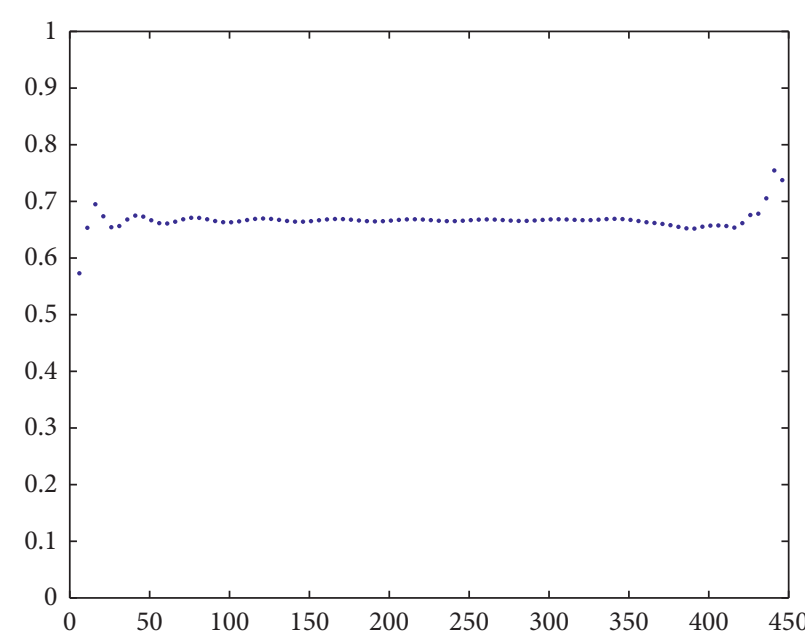

(a)

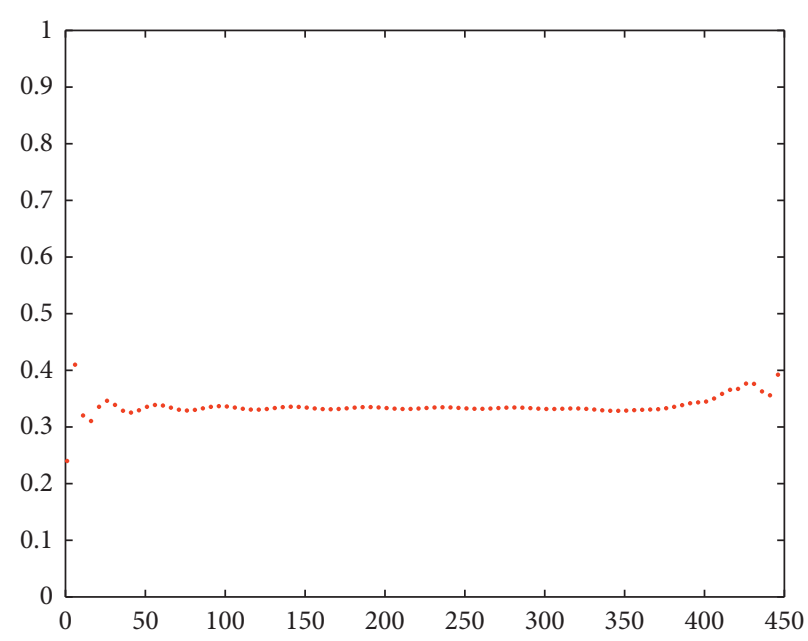

(b)

Figure 1: The stable behavior of the prey and predator populations for Example 1 to the equilibrium population points. (a) The graph on $p_{1}-t$ plane of Example 1. (b) The graph on $p_{2}-t$ plane of Example 1.

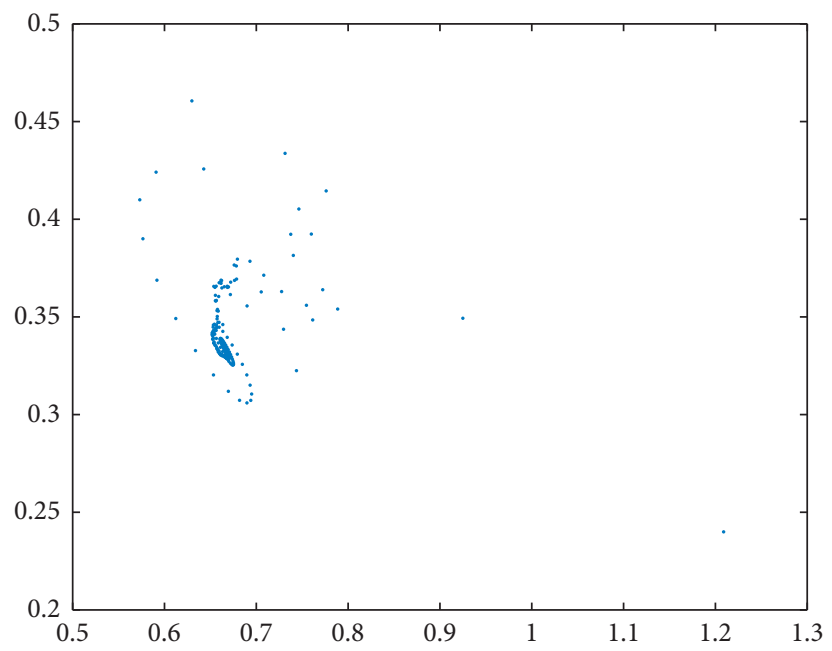

FIGURE 2: The phase graph of Example 1 in $t=500$ with the equilibrium points $p_{1}^{*}=(2 / 3)$ and $p_{2}^{*}=(1 / 3)$.

TABle 1: Errors for Example 2.

\begin{tabular}{|c|c|c|c|c|c|c|}
\hline \multirow{3}{*}{$t$} & \multicolumn{3}{|c|}{$R_{1, N}(t)$} & \multicolumn{3}{|c|}{$R_{2, N}(t)$} \\
\hline & \multicolumn{2}{|c|}{ Present method } & \multirow{2}{*}{$\begin{array}{l}\text { Method in [23] } \\
\qquad N=5\end{array}$} & \multicolumn{2}{|c|}{ Present method } & \multirow{2}{*}{$\begin{array}{c}\text { Method in [23] } \\
\qquad N=5\end{array}$} \\
\hline & $N=5$ & $N=12$ & & $N=5$ & $N=12$ & \\
\hline 0.0 & $5.88378 e-8$ & $3.56178 e-15$ & $\simeq\left[10^{-5}, 10^{-10}\right]$ & $3.415153 e-8$ & $8.881278 e-15$ & $\simeq\left[10^{-5}, 10^{-10}\right]$ \\
\hline 0.1 & $5.76570 e-7$ & $1.76292 e-14$ & $\simeq\left[10^{-5}, 10^{-10}\right]$ & $1.45283 e-7$ & $8.743266 e-14$ & $\simeq\left[10^{-5}, 10^{-10}\right]$ \\
\hline 0.2 & $1.36470 e-6$ & $2.87548 e-14$ & $\simeq\left[10^{-5}, 10^{-10}\right]$ & $2.87360 e-8$ & $1.65978 e-14$ & $\simeq\left[10^{-5}, 10^{-10}\right]$ \\
\hline 0.3 & $3.65207 e-8$ & $4.27880 e-12$ & $\simeq\left[10^{-5}, 10^{-10}\right]$ & $3.61146 e-8$ & $2.37310 e-13$ & $\simeq\left[10^{-5}, 10^{-10}\right]$ \\
\hline 0.4 & $6.42077 e-6$ & $3.62315 e-12$ & $\simeq\left[10^{-5}, 10^{-10}\right]$ & $1.54657 e-7$ & $2.02147 e-13$ & $\simeq\left[10^{-5}, 10^{-10}\right]$ \\
\hline 0.5 & $4.04666 e-6$ & $5.97320 e-12$ & $\simeq\left[10^{-5}, 10^{-10}\right]$ & $5.76562 e-7$ & $4.63123 e-13$ & $\simeq\left[10^{-5}, 10^{-10}\right]$ \\
\hline 0.6 & $2.37656 e-6$ & $7.28578 e-12$ & $\simeq\left[10^{-5}, 10^{-10}\right]$ & $4.63832 e-7$ & $5.21534 e-13$ & $\simeq\left[10^{-5}, 10^{-10}\right]$ \\
\hline 0.7 & $3.34357 e-6$ & $7.51433 e-12$ & $\simeq\left[10^{-5}, 10^{-10}\right]$ & $5.66931 e-7$ & $5.75760 e-13$ & $\simeq\left[10^{-5}, 10^{-10}\right]$ \\
\hline 0.8 & $2.21441 e-5$ & $1.99510 e-12$ & $\simeq\left[10^{-5}, 10^{-10}\right]$ & $3.65471 e-6$ & $7.44423 e-12$ & $\simeq\left[10^{-5}, 10^{-10}\right]$ \\
\hline 0.9 & $3.27256 e-4$ & $5.44983 e-12$ & $\simeq\left[10^{-5}, 10^{-10}\right]$ & $2.44449 e-5$ & $4.42886 e-12$ & $\simeq\left[10^{-5}, 10^{-10}\right]$ \\
\hline 1.0 & $2.27723 e-5$ & $4.08768 e-12$ & $\simeq\left[10^{-5}, 10^{-10}\right]$ & $4.33326 e-5$ & $2.75555 e-10$ & $\simeq\left[10^{-5}, 10^{-10}\right]$ \\
\hline
\end{tabular}


TABLE 2: Comparison of the absolute errors obtained by different rates for $R_{1, N}$ in Example 3 .

\begin{tabular}{|c|c|c|c|c|c|c|}
\hline \multirow[t]{2}{*}{$t$} & \multicolumn{2}{|c|}{$\begin{array}{c}A=0.45, B=0.15 \\
C=0.45, D=0.9\end{array}$} & \multicolumn{2}{|c|}{$\begin{array}{l}A=0.8, B=0.4 \\
C=0.4, D=0.6\end{array}$} & \multicolumn{2}{|c|}{$\begin{array}{c}A=0.16, B=0.48 \\
C=0.3, D=0.15\end{array}$} \\
\hline & $N=7$ & $N=9$ & $N=7$ & $N=9$ & $N=7$ & $N=9$ \\
\hline 0.2 & $3.75745 e-12$ & $1.30547 e-14$ & $2.34442 e-11$ & $2.12354 e-13$ & $1.65098 e-11$ & $2.77903 e-15$ \\
\hline 0.4 & $1.00987 e-12$ & $3.22244 e-14$ & $1.02348 e-11$ & $6.77926 e-14$ & $1.30007 e-11$ & $5.99001 e-13$ \\
\hline 0.6 & $6.43322 e-13$ & $9.91158 e-15$ & $4.82091 e-13$ & $4.66258 e-15$ & $8.20989 e-13$ & $5.56193 e-15$ \\
\hline 0.8 & $4.49987 e-11$ & $9.00302 e-14$ & $3.35303 e-11$ & $6.39937 e-15$ & $8.96511 e-11$ & $4.09824 e-15$ \\
\hline 1.0 & $2.66561 e-10$ & $9.21367 e-14$ & $6.12546 e-11$ & $9.09012 e-15$ & $5.36768 e-11$ & $2.09891 e-15$ \\
\hline
\end{tabular}

TABle 3: Comparison of the absolute errors obtained by different rates for $R_{2, N}$ in Example 3.

\begin{tabular}{|c|c|c|c|c|c|c|}
\hline \multirow[t]{2}{*}{$t$} & \multicolumn{2}{|c|}{$\begin{array}{c}A=0.45, B=0.15 \\
C=0.45, D=0.9\end{array}$} & \multicolumn{2}{|c|}{$\begin{array}{l}A=0.8, B=0.4 \\
C=0.4, D=0.6\end{array}$} & \multicolumn{2}{|c|}{$\begin{array}{c}A=0.16, B=0.48 \\
C=0.3, D=0.15\end{array}$} \\
\hline & $N=7$ & $N=9$ & $N=7$ & $N=9$ & $N=7$ & $N=9$ \\
\hline 0.2 & $3.86301 e-11$ & $4.21008 e-15$ & $8.23171 e-11$ & $7.9878 e-15$ & $5.76173 e-11$ & $2.21378 e-15$ \\
\hline 0.4 & $2.45611 e-12$ & $9.59823 e-15$ & $8.06578 e-11$ & $3.28935 e-15$ & $7.20981 e-12$ & $4.98678 e-15$ \\
\hline 0.6 & $1.87501 e-12$ & $2.00256 e-15$ & $1.63809 e-12$ & $4.99876 e-15$ & $2.65410 e-12$ & $1.57792 e-15$ \\
\hline 0.8 & $2.12409 e-10$ & $1.55729 e-14$ & $8.28909 e-11$ & $1.37811 e-15$ & $2.00256 e-11$ & $3.32209 e-14$ \\
\hline 1.0 & $9.12781 e-10$ & $2.81009 e-14$ & $3.12927 e-10$ & $8.44606 e-15$ & $1.34690 e-10$ & $5.67813 e-14$ \\
\hline
\end{tabular}

Example 2. Consider the Lotka-Volterra predator-prey models with discrete delay given in [23] by

$$
\left\{\begin{array}{l}
p_{1}^{\prime}(t)=p_{1}(t)\left[1-p_{2}(t)\right], \\
p_{2}^{\prime}(t)=p_{2}(t)\left[-1+p_{1}(t)\right],
\end{array}\right.
$$

with initial conditions

$$
\begin{aligned}
& p_{1}(0)=1.3, \\
& p_{2}(0)=0.6 .
\end{aligned}
$$

We selected our second example from [23], which solved this predator-prey interaction by the Bessel collocation approach. We compared our results with the Bessel collocation results, and the outcomes are tabulated in Table 1. For this example too, the improved Collocation approach of Euler polynomials connected with Bernoulli ones is incisive. This is because for the same number of basis functions, it obtains better results. Table 1 shows the present method results for Example 2 in comparison with method of [23]. The superiority of Euler polynomials operational matrices method compared with Bessel collocation approach is clear here, which is because with the same number of basis functions, we get very better results.

Example 3. Suppose in a closed ecosystem there are only 2 types of animals: the predator and the prey. They form a simple food-chain where the predator species hunts the prey species, while the prey grazes vegetation. The size of the 2 populations can be described by a simple system of 2 nonlinear first-order differential equations:

$$
\left\{\begin{array}{l}
p_{1}^{\prime}(t)=p_{1}(t)\left[A-B p_{2}(t)\right], \\
p_{2}^{\prime}(t)=p_{2}(t)\left[-C+D p_{1}(t)\right],
\end{array}\right.
$$

which a set of fixed positive constants. $A$ : the growth rate of the prey, $B$ : the rate at which predators destroy the prey, $C$ : the death rate of predators, and $D$ : the rate at which predators increase by consuming the prey.

A prey population $p_{1}(t)$ increases at a rate $\left(\mathrm{d} p_{1}(t) / \mathrm{d} t\right)=$ $A p_{1}(t)$ (proportional to the number of prey) but is simultaneously destroyed by predators at a rate $\left(\mathrm{d} p_{1}(t) / \mathrm{d} t\right)=$ $-B p_{1}(t) p_{2}(t)$ (proportional to the product of the number of prey and predators). A predator population $p_{2}(t)$ decreases at a rate $\left(\mathrm{d} p_{2}(t) / \mathrm{d} t\right)=-C p_{2}(t)$ (proportional to the number of predators), but increases at a rate $\left(\mathrm{d} p_{2}(t) / \mathrm{d} t\right)=$ $D p_{1}(t) p_{2}(t)$ (again proportional to the product of the numbers of prey and predators). By Theorem 1 , we have the the positive equilibrium $E^{*}=\left(p_{1}^{*}=(C / D), p_{2}^{*}=(A / B)\right)$.

For this example, the stability of each of the three steady states can be assessed more formally using the approach discussed. The absolute errors for $N=7,9$ are estimated by $R_{1, N}$ and $R_{2, N}$ and are presented in Tables 2 and 3 . We see that if $\mathrm{N}$ increases, then the absolute errors decrease more rapidly.

\section{Conclusion}

In this paper, we have proposed a numerical approach for solving the Lotka-Volterra predator-prey models with discrete delay by utilizing Euler polynomials connected with Bernoulli ones. The cognate matrices and mixed-product property of Kronecker products, besides the collocation method, have been utilized for transforming a predator-prey system to a linear system of algebraic equations that can be solved facilely. To the best of our cognizance, this is the first work coalescing the Euler polynomial connected with the Bernoulli polynomial and collocation points for solving Lotka-Volterra predator-prey models. Finally, numerical examples reveal that the present method is very precise and convenient for solving predator-prey models. 


\section{Data Availability}

No data were used to support this study.

\section{Conflicts of Interest}

The authors declare that they have no conflicts of interest.

\section{References}

[1] N. H. Aljahdaly and M. A. Alqudah, "Analytical solutions of a modified predator-prey model through a new ecological interaction," Computational and Mathematical Methods in Medicine, vol. 2019, Article ID 4849393, 7 pages, 2019.

[2] A. Das and M. Pal, "Theoretical analysis of an imprecise preypredator model with harvesting and optimal control," Journal of Optimization, vol. 2019, Article ID 9512879, 12 pages, 2019.

[3] Q. Han, W. Xu, B. Hu, D. Huang, and J.-Q. Sun, "Extinction time of a stochastic predator-prey model by the generalized cell mapping method," Physica A: Statistical Mechanics and Its Applications, vol. 494, pp. 351-366, 2018.

[4] C. Leal-Ramírez, O. Castillo, P. Melin, and H. EchavarriaHeras, "A fuzzy cellular prey-predator model for pest control under sustainable bio-economic equilibrium: a formal description and simulation analysis study," Applied Mathematical Modelling, vol. 39, no. 7, pp. 1794-1803, 2015.

[5] S. Ruan, "On nonlinear dynamics of predator-prey models with discrete delay," Mathematical Modelling of Natural Phenomena, vol. 4, no. 2, pp. 140-188, 2009.

[6] M. Umar, Z. Sabir, and M. A. Z. Raja, "Intelligent computing for numerical treatment of nonlinear prey-predator models," Applied Soft Computing, vol. 80, pp. 506-524, 2019.

[7] V. Volterra and M. Brelot, Leçons sur la théorie mathématique de la lutte pour la vie, Paris: Gauthier-Villars, Paris, France, 1931.

[8] M. Brelot, "Sur le problème biologique héréditaire de deux espèces dévorante et dévorée," Annali di Matematica Pura ed Applicata, vol. 9, no. 1, pp. 57-74, 1931.

[9] J. M. Cushing, Integrodifferential Equations and Delay Models in Population Dynamics, Vol. 20, Springer Science \& Business Media, Berlin, Germany, 2013.

[10] N. Apreutesei and G. Dimitriu, "On a prey-predator reactiondiffusion system with holling type iii functional response," Journal of Computational and Applied Mathematics, vol. 235, no. 2, pp. 366-379, 2010.

[11] M. R. Garvie, "Finite-difference schemes for reaction-diffusion equations modeling predator-prey interactions in MATLAB," Bulletin of Mathematical Biology, vol. 69, no. 3, pp. 931-956, 2007.

[12] M. R. Garvie and C. Trenchea, "Finite element approximation of spatially extended predator-prey interactions with the holling type II functional response," Numerische Mathematik, vol. 107, no. 4, pp. 641-667, 2007.

[13] S. Ruan, "Absolute stability, conditional stability and bifurcation in Kolmogorov-type predator-prey systems with discrete delays," Quarterly of Applied Mathematics, vol. 59, no. 1, pp. 159-173, 2001.

[14] X.-Z. He, "Stability and delays in a predator-prey system," Journal of Mathematical Analysis and Applications, vol. 198, no. 2, pp. 355-370, 1996.

[15] Z. Lu and W. Wang, "Global stability for two-species LotkaVolterra systems with delay," Journal of Mathematical Analysis and Applications, vol. 208, no. 1, pp. 277-280, 1997.
[16] T. Faria, "Stability and bifurcation for a delayed predator-prey model and the effect of diffusion," Journal of Mathematical Analysis and Applications, vol. 254, no. 2, pp. 433-463, 2001.

[17] P. T. Young, "Congruences for Bernoulli, euler, and stirling numbers," Journal of Number Theory, vol. 78, no. 2, pp. 204-227, 1999.

[18] F. Mirzaee and S. Bimesl, "A uniformly convergent euler matrix method for telegraph equations having constant coefficients," Mediterranean Journal of Mathematics, vol. 13, no. 1, pp. $497-515,2016$

[19] F. Mirzaee and N. Samadyar, "Explicit representation of orthonormal Bernoulli polynomials and its application for solving Volterra-Fredholm-Hammerstein integral equations," SeMA Journal, vol. 77, no. 1, pp. 81-96, 2019.

[20] F. Mirzaee, N. Samadyar, and S. F. Hoseini, "Euler polynomial solutions of nonlinear stochastic Itô-Volterra integral equations," Journal of Computational and Applied Mathematics, vol. 330, pp. 574-585, 2018.

[21] G.-S. Cheon, "A note on the Bernoulli and Euler polynomials," Applied Mathematics Letters, vol. 16, no. 3, pp. 365368, 2003.

[22] X.-P. Yan and Y.-D. Chu, "Stability and bifurcation analysis for a delayed Lotka-Volterra predator-prey system," Journal of Computational and Applied Mathematics, vol. 196, no. 1, pp. 198-210, 2006.

[23] Ş. Yüzbaşı, "Bessel collocation approach for solving continuous population models for single and interacting species," Applied Mathematical Modelling, vol. 36, no. 8, pp. 37873802, 2012. 